\title{
Surgical Treatment of Lumbar Hyperextension Injury in Ankylosing Spondylitis
}

\author{
Jung Keun Lee, Ki Seok Park, Moon Sun Park, Seong Min Kim, \\ Seung Young Chung, Do Sung Lee
}

\author{
Department of Neurosurgery, Eulji University College of Medicine, Taejon, Korea
}

Ankylosing spondylitis (AS) is a chronic systemic and inflammatory rheumatic disease with a variable course of the axial skeleton. Spinal involvement may accompany ossification of the ligaments, intervertebral disc, end-plates and apophyseal structures, and seems to be "bamboo spine". Because of these natures of the spine in AS, a spinal fracture can be occurred with minor trauma or spontaneously. The fracture of the AS can cause neurological complications extremely high, so special attention to prevent neurological deterioration. Operative management of the injured spine with AS is difficult, and associated with a high complication rate. Extreme care must be taken for surgery to prevent secondary neurological deterioration.

Key Words: Ankylosing spondylitis $\cdot$ Lumbar fracture $\cdot$ Postoperative complication $\cdot$ Neurological deterioration

\section{INTRODUCTION}

Ankylosing spondylitis (AS) is a chronic inflammatory disease which is characterized by pain and progressive stiffness, which could mainly affect spinal columns and sacroiliac joints ${ }^{3)}$. The sacroiliac joints and lumbar spines are most commonly affected, but with advanced disease, the entire spinal column may be involved ${ }^{5)}$. AS is strongly associated with the HLA-B27 gene; $90-95 \%$ of patients with AS are positive for HLA-B27 ${ }^{1}$. The prevalence of AS ranges between $0.2 \%$ and $0.55 \%$, and the male to female ratio is $2.5: 1^{1)}$.

An autoimmune response leads to the fibrosis and ossification of ligaments and joints of the spine, and in the end-stage to an individual and disseminated auto fusion of the spinal segments ${ }^{5}$. This process could lead to syndesmophytes of the disc, alter vertebral body formation, and cause to be the appearance of the bamboo spine. The rigid spinal segments present a long lever arm, which is unable to hold up the energy of a fall ${ }^{1)}$. In addition, it has been established that osteoporotic change is occurred early in the $\mathrm{AS}^{6}$. Resulting kyphosis, spinal rigidity and secondary osteoporosis lead to changes of the biomechanical characteristics of the spinal column ${ }^{6,10}$. Secondary

- Received: July 14, 2013 • Revised: September 12, 2013

- Accepted: September 14, 2013

Corresponding Author: Ki Seok Park, MD

Department of Neurosurgery, Eulji University Hospital, 1306 Dunsan-

dong, Seo-gu, Daejeon 302-799, Korea

Tel: +82-42-611-3442, Fax: +82-42-611-3444

E-mail: ks3432@eulji.ac.kr to kyphosis, the ventral displacement creates pathological tension and shearing forces to a spine with lacking flexibility ${ }^{20)}$. Furthermore, the reduced muscle activity and increased muscle degeneration causes an overall loss of muscle strength ${ }^{18)}$. The resulting posture change, with its pathologically elongated lever arms and reduced bone quality, can lead to serious injuries even after minor trauma mechanisms ${ }^{6}$. Frequently, no trauma is recalled by the patient, but rather an abrupt motion or other inadequate mechanism ${ }^{6}$. Especially if a complete rigidity of the spine is present, there is an increasing risk sustaining such injuries ${ }^{18)}$. In these cases, the rate of neurological complications is extremely high, and special attention must be addressed to delayed or secondary neurological complications.

Initial immobilization in the patient's preinjury alignment is mandatory to prevent secondary neurologic injury. Both nonoperative and operative treatments can be employed depending on the patient's age, comorbidities, and fracture stability $^{13)}$. Preoperative management of the injured spine with AS is difficult and technically challenging due to the osteoporotic changes, stiffness, and cage subsidence, in addition to pseudoarthrosis ${ }^{9,11,16}$.

In this case, we report a case having a surgical treatment with lumbar fracture injury in AS.

\section{CASE REPORT}

A 43-year-old man was admitted for severe low back pain after slipped down on the icy roads. He had a history of AS. 
Findings from the physical examination consisted in tenderness to palpation of the lumbar spine at L1. The neurological findings were normal. Plain radiographs of the lumbar spine revealed a bamboo spine typical for AS, with a visible fracture line in the L1-2 spine. A whole spine computed tomography (CT) confirmed the presence of a transcorporeal bony fracture of the L2 lumbar spine. By magnetic resonance imaging (MRI), the T2 sequence disclosed high signal intensity from the body and neural arch of L2, consistent with a recent fracture. MRI also demonstrated injury of posterior interspinous ligaments and there was no thecal compression and cord signal change (Fig. 1). This fracture in a patient with AS involved all three spinal columns and was therefore considered unstable. We decided to perform the surgical treatment due to highly unstable fracture condition.

Preoperative evaluation was done for general anesthesia. He had cardiovascular and pulmonary disease; aortic regurgitation, mitral valve disease and fibrous disease in the lung
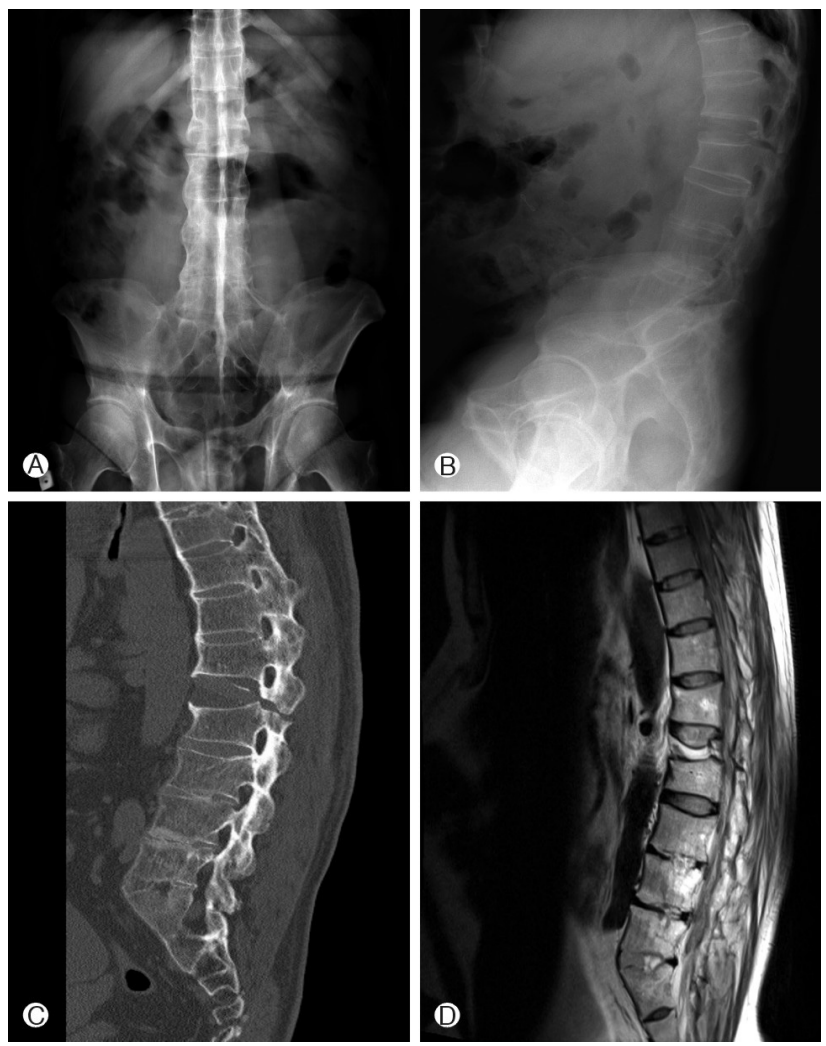

Fig. 1. Preoperative images of the patient. A 42-year-old male AS patient slipped down on icy roads and suffered from an unstable L2 burst fracture. (A) Anterioposterior plain radiography image which demonstrate L2 burst fracutre and bamboo spine. (B) Lateral plain radiography image which demonstrate $L 2$ burst fracutre and bamboo spine. (C) Lateral image on computed tomography showes transcorporeal fracture. (D) T2 magnetic resonance image showes three column injury. apex. We had to consider the patient's medical disease. When performing intubation, we did it fiberoptically in the awakening state due to typical kyphotic bamboo spine.

At first, we performed the posterior fixation of T12, L1, L2, L3. Post posterior surgical management, the disalignment was detected on L1-2 in the plain radiography. After that, we did anterior approach using the expendable cage. Performing combined posterior and anterior approach, the patient showed left lower extremity weakness in hip flexion of grade 2 and left knee extension of grade 1. From the retrospective review, we noticed that disalignment at the fracture site was happened in prone positioning for posterior fixation. Postoperative computed tomography revealed that the left $\mathrm{L} 2$ root was compressed between disaligned lamina and pedicle (Fig. 2). The patient was put under absolute bed rest and administrated intravenous steroid. On the first postoperative day, we performed root decompression and the patient showed improvement on the neurologic examination as the motor grade 3 of Lt. knee extension and hip flexion. After 2 days of absolute bed rest, the patient received rehabilitation treatment with the aid of orthosis for two months. On the follow-up radiographs obtained 1 and 2 months after surgery, the cage subsidence was shown gradually. But the cage subsidence was not aggravated after the last 2 months radiographs.

\section{DISCUSSION}

As outlined in this case report, the special attention is required in patients with suspected spinal fractures with AS. The rigidity of the spine, the resulting kyphotic deformity, the associated osteoporosis, and the degenerated stabilizing muscles lead to increased vulnerability of the spinal column ${ }^{19}$. The rigid spinal segments and osteopenia influence on the evident pathologic kyphotic posture and the spinal fragility ${ }^{6}$. Therefore, a meticulous screening of the entire spine is necessary in these patients with new onset or increased pain spontaneously and after minor trauma ${ }^{14)}$. The fractures with AS occurred mostly through the interspace of vertebral bodies rather than the vertebral body itself ${ }^{13)}$. The patients who were diagnosed with completely ankylosed spine at an average age of 50 years undergo more severe fractures than the others ${ }^{18)}$.

When a spinal fracture occurs, it may be extremely unstable with an associated high risk of neurological deficit (range from $69 \%$ to $75 \%)^{18)}$. The risk for neurological deficit has been reported in up to $57 \%$ of patients with AS. Spinal fracture risk is increased 4-fold in AS patients compared to the general population, and $5 \%$ to $15 \%$ of all AS patients experience a spinal fracture at some point during their life ${ }^{7}$. The neurological deficit may occur acutely or be delayed due to 

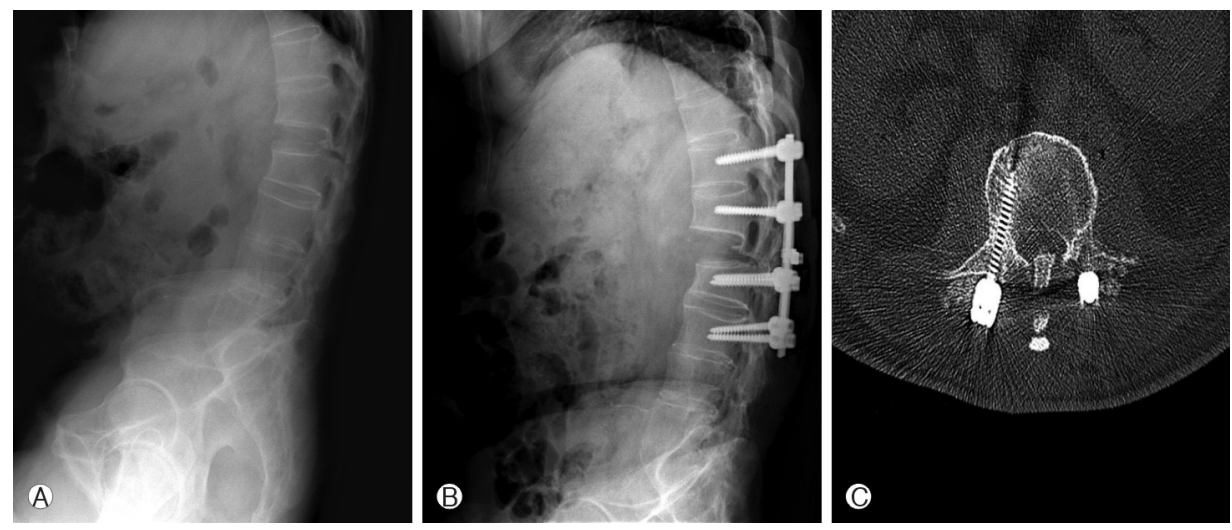

Fig. 2. Postoperative complication after posterolateral fusion. (A) Preoperative lateral pain radiography image. (B) Postoperative lateral pain radiography image. At the point of circle, spinal cord was compressed by posterior column and L1-2 intervertebral space gap was increased. (C) On postoperative computed tomo- graphy axial image, bilateral lamina and spinous process compressed spinal cord and nerve roots.
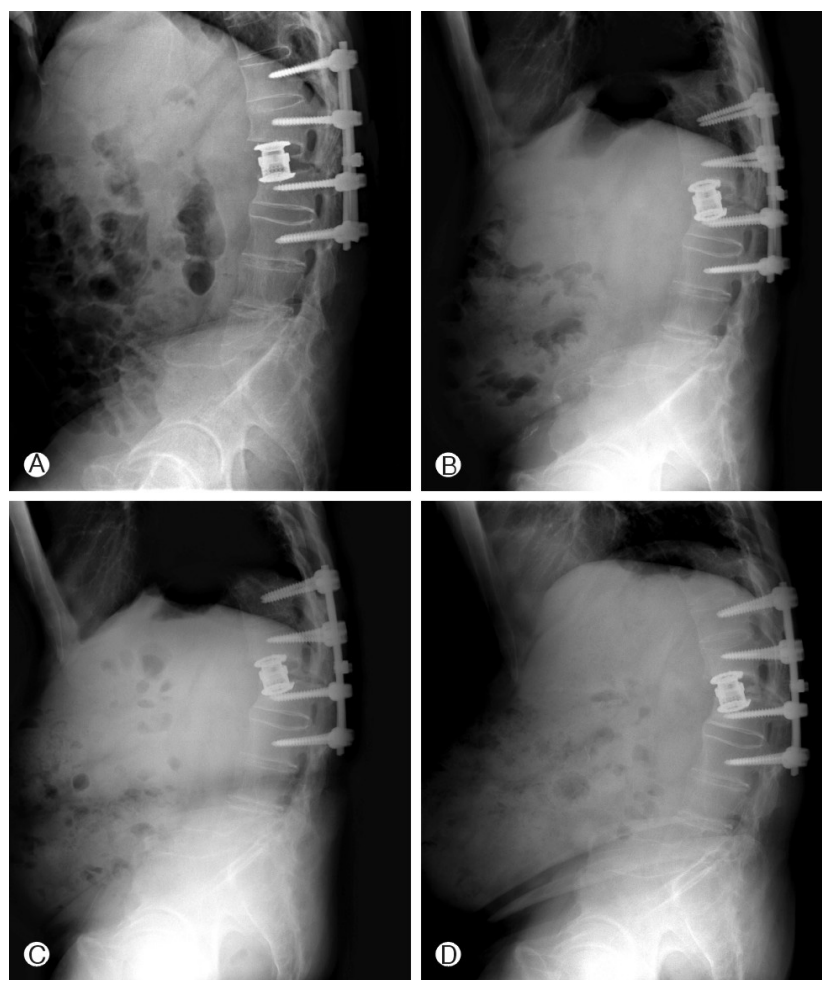

Fig. 3. Follow up images after postero-anterior stabilization. (A) Immediate postoperative lateral plain radiography image. (B) 1 month later lateral plain radiography image. (C) 2 month later lateral plain radiography image. Cage subsidence on up and down L1, 2 vertebral body was identified, which changes was occurred due to the nature of AS spines, but no neurologic deficit. (D) 1 year later lateral plain radiography images. cage subsidence is not advanced until now.

spinal instability. It can be more generated by a slipped disc, an avulsion fracture or an epidural hematoma ${ }^{2}$. Farhal et al proposed that cancellous bone in patients with AS may be more likely to bleed following a fracture, so it can cause to be an epidural hematomas).

The radiological imaging of spine fractures in patients with AS demands special attention since these injuries are frequently missed and delayed in their diagnosis ${ }^{6}$. But spinal fractures in AS patients can be difficult to identify with standard X-rays due to spinal ossification, osteoporosis, poor visibility of the disc spaces and the difficulty of exploring certain regions of the spine, especially the cervicothoracic region ${ }^{17}$. The proportion of fractures that are missed initially ranges from 19\% to $60 \%{ }^{122}$. These diagnostic delays explain the up to $15 \%$ rate of secondary neurological complications before fracture treatment ${ }^{13)}$. So many authors recommend to check the whole spine using a CT-scan with multiplanar reconstruction for detecting occult fractures ${ }^{12}$.

The main goal of treatment is the stabilization of the fracture in the correct position until complete fracture healing is achieved. Whether operative or non-operative management, the treatment is determined in terms of the stability of the fracture and the existence of neurological signs ${ }^{14}$. Non-operative treatment options are external orthoses, traction, halovest management. Non-operative treatment can be used in patients with non-displaced fractures ${ }^{13)}$. However, the unyielding kyphotic deformity makes bracing difficult, and surgery is therefore often required to treat these unstable fractures. Indications for operative treatment are based on the criteria for instability, incorrect posture, pain and neurological defi$\mathrm{cit}^{15)}$. Many treatment strategies have been proposed in the literature, including anterior, posterior or combined anteriorposterior fixation. Isolated anterior stabilizations are rarely indicated $^{15)}$. The frequently recommended posterior instrumentation results in higher stability opposed to the single ven- 
tral stabilization ${ }^{20)}$. The combined posterior-anterior or anterior-posterior stabilizations are recommended due to resulting higher primary stability ${ }^{13,15)}$. So, in our case, we performed combined posterior-anterior approaches.

Generally, surgical treatment in prone position of spinal fractures can help to reduce the danger of neurological deterioration and allow a first reduction and stabilization in a safe way $^{20)}$. But patients with AS are at increased risk for posture deterioration and iatrogenic fractures of the spine during the surgical procedure, especially while under sedation and anesthesia ${ }^{11)}$. Extreme care must be taken when moving or lifting patients or during positioning for surgery to maintain fixed spinal kyphosis and to prevent fracture or dislocation ${ }^{15)}$. In our case, when positioning, the displacement was happened, so the patient showed secondary neurological deterioration after operation. This positioning is highly demanding and must take place under secured protection of the spine, and final positioning of the spine must be performed with the use of lateral spine fluoroscopy ${ }^{11)}$. In the reports, the acute onset or progression of neurological deficits had been reported after emergency intubations). Patients with AS are at high risk of these complications, standardized managements is required in patients including fiberoptical intubation, appropriate transport maneuvers and positioning under the avoidance of any brusque manipulation ${ }^{8)}$. A stable and safe surgical stabilization can allow the patients to avoid secondary neurological complications and do immediate postoperative mobilization.

Spine surgery in patients with AS is more difficult due to the fragility of the spinal column. Especially, due to spinal rigidity and osteoporotic change, cage subsidence and pseudoarthrosis could be unbroken in AS. We used the expendable cage which seems useful in vertebral body replacement in patients with osteoporotic thoracolumbar collapse ${ }^{17}$. Uchida et al suggested the expandable cage fits into the fusion site because of its in situ lengthening and there is no need to trim the cage. The proper fit of the expandable cage may create biomechanically and biologically appropriate conditions at the fusion site. Moreover, such operative management would be expected to reduce the rate of cage subsidence and implant dislodgement ${ }^{17}$. But cage subsidence was happened in our patients on follow up plain radiographs. In other reports, cage subsidence in vertebral bodies relies heavily on cancellous bone density which was most severely affected by osteoporosis ${ }^{9}$. March $\mathrm{L}$ et al. reported that on the osteoporotic vertebral body, the peripheral regions of the endplate have greater resistance to subsidence, whereas the central region provides the least resistance ${ }^{9}$. So grafted autobone inside and around the wide cages (more than $22 \mathrm{~mm}$ ) is likely to be advantage for bony union due to adequate stress transmission and proper blood flow ${ }^{16}$. Although, grafted autobone itself is already changed weakly, which can help prevent to some degrees of cage subsidence ${ }^{16}$. There are some possible causes for the formation of a pseudoarthrosis ${ }^{4}$. First, the segment in question may have escaped fusion while other levels became ossified. The process of spinal ossification in AS is multifocal and not contiguous, so this process may leave short mobile segments between long ankylosed segments. This sets the scene for high stress and mechanical failure. Secondly, the mechanics of a stiff kyphotic spine must result in high stresses, and repeated stress may lead to fatigue fracture as in stress fractures of the long bones. These stress fractures would be more likely to occur through an ankylosing disc than the vertebral body itself due to less resistance of disc.

Finally, extraskeletal disease involves multiple organ systems, with each subtype of spondyloarthropathy having a somewhat different predilection for each. Such disease includes cardiovascular disease, more specifically aortic insufficiency and conduction disturbance; pulmonary disease, such as apical pulmonary fibrosis; deposition disease, such as amyloidosis with its associated renal dysfunction ${ }^{5)}$. As above mentioned, our patient had aortic regurgitation, mitral valve disease and fibrous disease in the lung apex. It is important that these medical complicating problems with AS should be carefully considered before operation to do a safety surgery and get the good outcome.

\section{CONCLUSION}

AS transforms the flexible spinal column into a stiff rod. Due to these spinal fragilities, spinal fractures in patients with AS can occur after minor injuries. The whole spine evaluation must be carried out even though patients primary complains are back pain due to neurologic deficit is not always accompanied and high risk of secondary neurologic deterioration. The primary aim of surgical treatment is the maintenance of fracture realignment with adequate stabilization measures until the bone has healed completely. Many complications can be accompanied such as secondary neurologic deficit, cage subsidence and pseudoarthrosis. We must do surgical treatment carefully considering the nature of AS and utmost care should be paid for avoiding neurologic deficit when lifting or during positioning for surgery.

\section{REFERENCES}

1. Braun J, Sieper J: Ankylosing spondylitis. Lancet. 369:13791390, 2007

2. Broom M, Raycroft JF: Complications of fractures of the cervical spine in ankylosing spondylitis. Spine 13:763-766, 1988 
3. Claire M, Andrew P: Diagnosis and management of ankylosing spondylitis. BMJ. 333:581-585, 2006

4. David F, John C, Leong Y, Eric K, Chan F, Chow S: Spinal pseudoarthrosis in ankysoling spondylitis. J Bone Joint Surg 70-B:443-447, 1988

5. Fox MW, Burton M, Burton OM, Kilgore JE: Neurological complications of ankylosing spondylitis. J Neurosurg 78:871878, 1993

6. Geusens P, Vosse D, Linden S: Osteoporosis and vertebral fractures in ankylosing spondylitis. Curr Opin Rheumatol 19:335339, 2007

7. Hitchon PW, From AM, Brenton MD, Glaser JA, Torner JC: Fractures of the thoracolumbar spine complicating ankylosing spondylitis. J Neurosurg 97:218-222, 2002

8. Lin BC, Chen IH: Anesthaesia for ankylosing spondylitis patients undergoing transpedicle vertebrectomy. Acta Anaesthesiol Sin 37:73-78, 1999

9. Marchi L, Abdala N, Oliveira L, Amaral R, Coutinho E, Pimenta L: Radiographic and clinical evaluation of cage subsidence after stand-alone lateral interbody fusion: J Neurosurg Spine. Jul 19: 110-118, 2013

10. Metz-Stavenhagen P, Krebs S, Meier O: Cervical fracture in conjunction with spondylitis ankylosans. Orthopade 30:925-931, 2001

11. Michael R, Sascha R, Christiane P, Harry R, Juergen H: Iatrogenic fractures in ankylosing spondylitis - a report of two cases. Eur Spine J 15(1):100-104, 2006

12. Milicic A, Jovanovic A, Milankov M, Savic D, Stankovic M: Fractures of the spine in patients with ankylosing spondylitis.
Med Pregl 48:429-431, 1995

13. Rowed DW: Management of cervical spinal cord injury in ankylosing spondylitis: the intervertebral disc as a cause of cord compression. J Neurosurg. Aug 77(2):241-246, 1992

14. Samartzis D, Anderson G, Shen F: Multiple and simultaneous spine fractures in ankylosing spondylitis. Spine 30:E711-E715, 2005

15. Shen FH, Samartzis D: Surgical management of lower cervical spine fracture in ankylosing spondylitis. J Trauma 61:10051009, 2006

16. Suzuki T, Abe E, Miyakoshi N, Murai H, Kobayashi T, Abe T, et al: Posterior-approach vertebral replacement with rectangular parallelepiped cages (PAVREC) for the treatment of osteoporotic vertebral collapse with neurological deficits. J Spinal Disord Tech. Jul 26(5):E170-E176, 2013

17. Uchida K, Kobayashi S, Nakajima H, Kokubo Y, Yayama T, Sato R et al: Anterior expandable strut cage replacement for osteoporotic thoracolumbar vertebral collapse. J Neurosurg Spine. Jun 4(6):454-462, 2006

18. Vosse D, Feldtkeller E, Erlendsson J, Geusens P, Linden S: Clinical vertebral fractures in patients with ankylosing spondylitis. J Rheumatol 31:1981-1985, 2004

19. Wang YF, Teng MM, Chang CY, Wu HT, Wang ST: Imaging manifestations of spinal fractures in ankylosing spondylitis. AJNR Am J Neuroradiol 26:2067-2076, 2005

20. Zdichavsky M, Blauth M, Knop C, Lange U, Krettek C, Bastian L: Ankylosing spondylitis. Therapy and complications of 34 spine fractures. Chirurg 76:967-975, 2005 